S4 Text. Attractors obtained in the simulation of mutant phenotypes

\title{
Wild-Type
}

Attractor(s) with period 11

\begin{tabular}{llllllllllll}
\hline & & \multicolumn{10}{c}{ Attr. 1} \\
APC/C & 1 & 1 & 0 & 0 & 0 & 0 & 0 & 0 & 0 & 0 & 1 \\
KRP1 & 0 & 1 & 0 & 0 & 0 & 0 & 1 & 0 & 0 & 0 & 0 \\
CYCA2;3 & 0 & 0 & 0 & 0 & 0 & 0 & 1 & 1 & 1 & 1 & 1 \\
CDKB1;1 & 1 & 1 & 0 & 0 & 0 & 0 & 1 & 1 & 1 & 1 & 1 \\
CYCB1;1 & 0 & 0 & 0 & 0 & 0 & 0 & 1 & 1 & 1 & 1 & 1 \\
MYB3R1/4 & 1 & 0 & 0 & 0 & 0 & 0 & 1 & 1 & 1 & 1 & 1 \\
MYB77 & 0 & 0 & 0 & 0 & 0 & 1 & 1 & 1 & 0 & 0 & 0 \\
E2Fe & 0 & 0 & 0 & 1 & 1 & 1 & 1 & 1 & 1 & 0 & 0 \\
E2Fc & 1 & 1 & 0 & 0 & 1 & 1 & 0 & 1 & 1 & 1 & 1 \\
E2Fb & 0 & 0 & 0 & 0 & 1 & 1 & 1 & 1 & 0 & 0 & 0 \\
E2Fa & 0 & 0 & 0 & 1 & 1 & 1 & 1 & 0 & 0 & 0 & 0 \\
RBR & 1 & 1 & 0 & 0 & 0 & 0 & 0 & 1 & 1 & 1 & 1 \\
SCF & 0 & 0 & 0 & 0 & 0 & 1 & 1 & 1 & 1 & 1 & 1 \\
CYCD3;1 & 0 & 1 & 1 & 1 & 1 & 1 & 0 & 0 & 0 & 0 & 0 \\
\hline Freq. & & & & 7 & $100 \%$ & & & & \\
\hline
\end{tabular}


APC/C loss of function

\begin{tabular}{lc} 
& Fixed-point attractor(s) \\
\hline APC/C & Attr. 1 \\
KRP1 & 0 \\
CYCA2;3 & 0 \\
CDKB1;1 & 1 \\
CYCB1;1 & 1 \\
MYB3R1/4 & 1 \\
MYB77 & 1 \\
E2Fe & 0 \\
E2Fc & 0 \\
E2Fb & 1 \\
E2Fa & 0 \\
RBR & 0 \\
SCF & 1 \\
CYCD3;1 & 1 \\
\hline Freq. & 0 \\
\hline
\end{tabular}


$\mathrm{APC} / \mathrm{C}$ gain of function

\begin{tabular}{llllllll}
\multicolumn{7}{c}{ Attractor(s) with period 7} \\
\hline APC/C & 1 & 1 & 1 & 1 & 1 & 1 & 1 \\
KRP1 & 0 & 0 & 0 & 1 & 1 & 1 & 1 \\
CYCA2;3 & 0 & 0 & 0 & 0 & 0 & 0 & 0 \\
CDKB1;1 & 0 & 0 & 0 & 1 & 1 & 1 & 1 \\
CYCB1;1 & 0 & 0 & 0 & 0 & 0 & 0 & 0 \\
MYB3R1/4 & 0 & 0 & 0 & 1 & 1 & 1 & 0 \\
MYB77 & 0 & 0 & 1 & 1 & 1 & 0 & 0 \\
E2Fe & 0 & 1 & 1 & 1 & 1 & 1 & 0 \\
E2Fc & 0 & 1 & 1 & 1 & 1 & 1 & 1 \\
E2Fb & 0 & 1 & 1 & 1 & 1 & 0 & 0 \\
E2Fa & 1 & 1 & 1 & 1 & 1 & 1 & 1 \\
RBR & 0 & 0 & 0 & 0 & 1 & 1 & 1 \\
SCF & 0 & 0 & 0 & 0 & 0 & 0 & 0 \\
CYCD3; & 1 & 1 & 1 & 1 & 1 & 1 & 1 \\
\hline Freq. & & & \multicolumn{7}{c}{$100 \%$} & & & \\
\hline
\end{tabular}




\section{KRP1 loss of function}

\begin{tabular}{|c|c|c|c|c|c|c|c|c|c|c|c|}
\hline \multirow[b]{2}{*}{$\mathrm{APC} / \mathrm{C}$} & \multicolumn{10}{|c|}{ Attractor(s) with period 11} & \\
\hline & 1 & 1 & 0 & 0 & 0 & 0 & 0 & 0 & 0 & 0 & 1 \\
\hline KRP1 & 0 & 0 & 0 & 0 & 0 & 0 & 0 & 0 & 0 & 0 & 0 \\
\hline CYCA $2 ; 3$ & 0 & 0 & 0 & 0 & 0 & 0 & 1 & 1 & 1 & 1 & 1 \\
\hline CDKB1;1 & 1 & 1 & 0 & 0 & 0 & 0 & 1 & 1 & 1 & 1 & 1 \\
\hline CYCB1;1 & 0 & 0 & 0 & 0 & 0 & 0 & 1 & 1 & 1 & 1 & 1 \\
\hline MYB3R1/4 & 1 & 0 & 0 & 0 & 0 & 0 & 1 & 1 & 1 & 1 & 1 \\
\hline MYB77 & 0 & 0 & 0 & 0 & 0 & 1 & 1 & 1 & 0 & 0 & 0 \\
\hline $\mathrm{E} 2 \mathrm{Fe}$ & 0 & 0 & 0 & 1 & 1 & 1 & 1 & 1 & 1 & 0 & 0 \\
\hline E2Fc & 1 & 1 & 0 & 0 & 1 & 1 & 0 & 1 & 1 & 1 & 1 \\
\hline $\mathrm{E} 2 \mathrm{Fb}$ & 0 & 0 & 0 & 0 & 1 & 1 & 1 & 1 & 0 & 0 & 0 \\
\hline $\mathrm{E} 2 \mathrm{Fa}$ & 0 & 0 & 0 & 1 & 1 & 1 & 1 & 0 & 0 & 0 & 0 \\
\hline RBR & 1 & 1 & 0 & 0 & 0 & 0 & 0 & 1 & 1 & 1 & 1 \\
\hline SCF & 0 & 0 & 0 & 0 & 0 & 1 & 1 & 1 & 1 & 1 & 1 \\
\hline CYCD3;1 & 0 & 1 & 1 & 1 & 1 & 1 & 0 & 0 & 0 & 0 & 0 \\
\hline Freq. & & & & & & $00^{6}$ & & & & & \\
\hline
\end{tabular}




\section{KRP1 gain of function}

\begin{tabular}{lcc}
\multicolumn{3}{c}{ Attractor(s) with period 2 } \\
\hline APC/C & \multicolumn{1}{c}{ Attr. 1 } \\
KRP1 & 0 & 1 \\
CYCA2;3 & 1 & 1 \\
CDKB1;1 & 0 & 0 \\
CYCB1;1 & 0 & 0 \\
MYB3R1/4 & 0 & 0 \\
MYB77 & 0 & 0 \\
E2Fe & 0 & 0 \\
E2Fc & 0 & 1 \\
E2Fb & 0 & 1 \\
E2Fa & 0 & 1 \\
RBR & 1 & 1 \\
SCF & 0 & 1 \\
CYCD3;1 & 0 & 0 \\
\hline Freq. & 1 & 1 \\
\hline
\end{tabular}


CYCA2;3 loss of function

\begin{tabular}{llllllll}
\multicolumn{7}{c}{ Attractor(s) with period 7} \\
\hline APC/C & 0 & 1 & 0 & 0 & 0 & 0 & 0 \\
KRP1 & 0 & 0 & 0 & 1 & 1 & 1 & 1 \\
CYCA2;3 & 0 & 0 & 0 & 0 & 0 & 0 & 0 \\
CDKB1;1 & 0 & 0 & 0 & 1 & 1 & 1 & 1 \\
CYCB1;1 & 0 & 0 & 0 & 1 & 1 & 1 & 1 \\
MYB3R1/4 & 0 & 0 & 0 & 1 & 0 & 1 & 0 \\
MYB77 & 0 & 0 & 1 & 0 & 1 & 0 & 0 \\
E2Fe & 0 & 1 & 1 & 1 & 1 & 1 & 1 \\
E2Fc & 0 & 1 & 0 & 1 & 1 & 0 & 1 \\
E2Fb & 0 & 1 & 0 & 1 & 1 & 0 & 1 \\
E2Fa & 1 & 1 & 1 & 1 & 1 & 1 & 1 \\
RBR & 0 & 1 & 0 & 0 & 1 & 0 & 1 \\
SCF & 0 & 0 & 0 & 0 & 1 & 0 & 1 \\
CYCD3;1 & 0 & 1 & 1 & 1 & 1 & 0 & 1 \\
\hline Freq. & & & \multicolumn{7}{c}{$100 \%$} & & \\
\hline
\end{tabular}


CYCA2;3 gain of function

\begin{tabular}{lccccccccccc}
\multicolumn{10}{c}{ Attractor(s) with period 11 } \\
\hline APC/C & 1 & 1 & 0 & 0 & 0 & 0 & 0 & 0 & 0 & 0 & 1 \\
KRP1 & 0 & 1 & 0 & 0 & 0 & 0 & 1 & 0 & 0 & 0 & 0 \\
CYCA2;3 & 1 & 1 & 1 & 1 & 1 & 1 & 1 & 1 & 1 & 1 & 1 \\
CDKB1;1 & 1 & 1 & 0 & 0 & 0 & 0 & 1 & 1 & 1 & 1 & 1 \\
CYCB1;1 & 0 & 0 & 0 & 0 & 0 & 0 & 1 & 1 & 1 & 1 & 1 \\
MYB3R1/4 & 1 & 0 & 0 & 0 & 0 & 0 & 1 & 1 & 1 & 1 & 1 \\
MYB77 & 0 & 0 & 0 & 0 & 0 & 1 & 1 & 1 & 0 & 0 & 0 \\
E2Fe & 0 & 0 & 0 & 1 & 1 & 1 & 1 & 1 & 1 & 0 & 0 \\
E2Fc & 1 & 1 & 0 & 0 & 1 & 1 & 0 & 1 & 1 & 1 & 1 \\
E2Fb & 0 & 0 & 0 & 0 & 1 & 1 & 1 & 1 & 0 & 0 & 0 \\
E2Fa & 0 & 0 & 0 & 1 & 1 & 1 & 1 & 0 & 0 & 0 & 0 \\
RBR & 1 & 1 & 0 & 0 & 0 & 0 & 0 & 1 & 1 & 1 & 1 \\
SCF & 0 & 0 & 0 & 0 & 0 & 1 & 1 & 1 & 1 & 1 & 1 \\
CYCD3;1 & 0 & 1 & 1 & 1 & 1 & 1 & 0 & 0 & 0 & 0 & 0 \\
\hline Freq. & & & 7 & $100 \%$ \\
\hline
\end{tabular}


CDKB1;1 loss of function

\begin{tabular}{llllllll}
\multicolumn{7}{c}{ Attractor(s) with period 7} \\
\hline APC/C & 0 & 1 & 0 & 0 & 0 & 0 & 0 \\
KRP1 & 0 & 0 & 0 & 1 & 1 & 1 & 1 \\
CYCA2;3 & 0 & 0 & 0 & 1 & 1 & 1 & 1 \\
CDKB1;1 & 0 & 0 & 0 & 0 & 0 & 0 & 0 \\
CYCB1;1 & 0 & 0 & 0 & 1 & 1 & 1 & 1 \\
MYB3R1/4 & 0 & 0 & 0 & 1 & 0 & 1 & 0 \\
MYB77 & 0 & 0 & 1 & 0 & 1 & 0 & 0 \\
E2Fe & 0 & 1 & 1 & 1 & 1 & 1 & 1 \\
E2Fc & 0 & 1 & 0 & 1 & 1 & 0 & 1 \\
E2Fb & 0 & 1 & 0 & 1 & 1 & 0 & 1 \\
E2Fa & 1 & 1 & 1 & 1 & 1 & 1 & 1 \\
RBR & 0 & 1 & 0 & 0 & 1 & 0 & 1 \\
SCF & 0 & 0 & 0 & 0 & 1 & 0 & 1 \\
CYCD3;1 & 0 & 1 & 1 & 1 & 1 & 0 & 1 \\
\hline Freq. & & & \multicolumn{7}{c}{$100 \%$} & & \\
\hline
\end{tabular}


CDKB1;1 gain of function

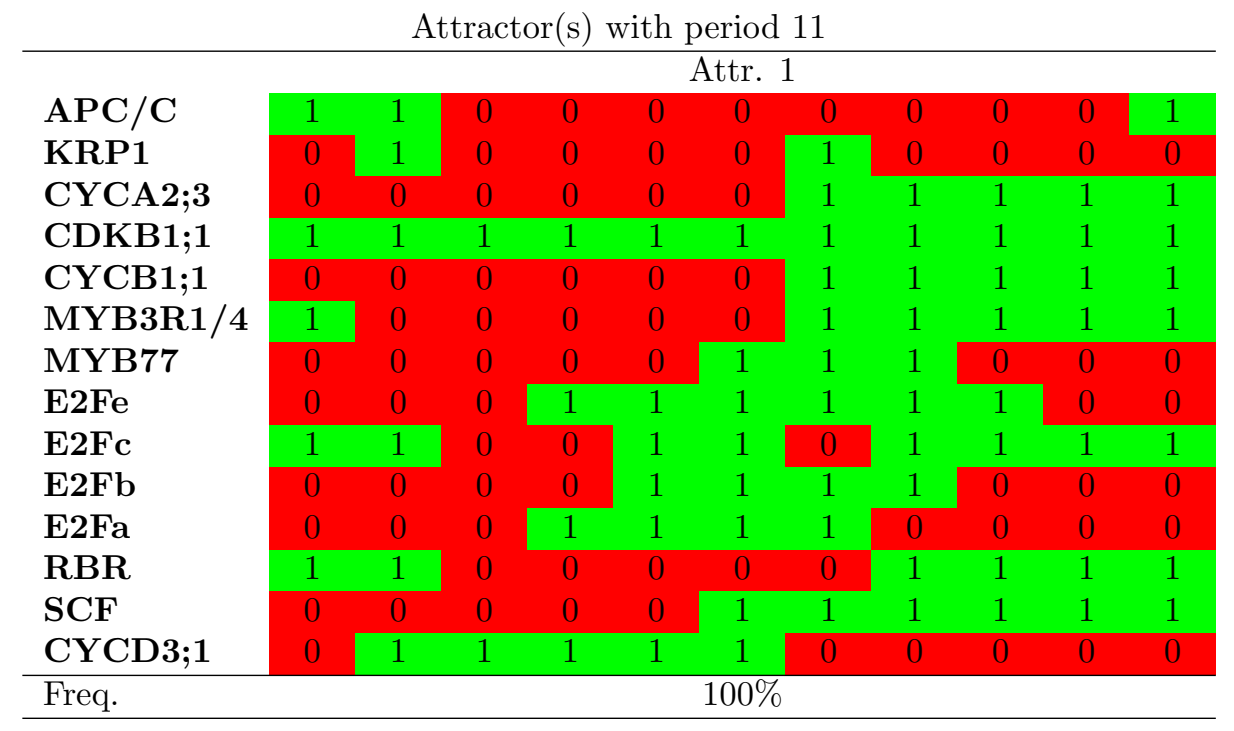


CYCB1;1 loss of function

\begin{tabular}{lllllllll}
\multicolumn{7}{c}{ Attractor(s) with period 8} \\
\hline APC/C & 0 & 0 & 0 & 0 & 0 & 0 & 0 & 0 \\
KRP1 & 0 & 0 & 0 & 0 & 1 & 0 & 0 & 0 \\
CYCA2;3 & 0 & 0 & 0 & 0 & 1 & 1 & 1 & 1 \\
CDKB1;1 & 0 & 0 & 0 & 0 & 1 & 1 & 1 & 1 \\
CYCB1;1 & 0 & 0 & 0 & 0 & 0 & 0 & 0 & 0 \\
MYB3R1/4 & 0 & 0 & 0 & 0 & 1 & 1 & 1 & 0 \\
MYB77 & 0 & 0 & 0 & 1 & 1 & 1 & 0 & 0 \\
E2Fe & 0 & 1 & 1 & 1 & 1 & 1 & 1 & 0 \\
E2Fc & 0 & 0 & 1 & 1 & 0 & 1 & 1 & 1 \\
E2Fb & 0 & 0 & 1 & 1 & 1 & 1 & 0 & 0 \\
E2Fa & 0 & 1 & 1 & 1 & 1 & 0 & 0 & 0 \\
RBR & 0 & 0 & 0 & 0 & 0 & 1 & 1 & 1 \\
SCF & 0 & 0 & 0 & 1 & 1 & 1 & 1 & 1 \\
CYCD3;1 & 0 & 1 & 1 & 1 & 0 & 0 & 0 & 0 \\
\hline Freq. & & & \multicolumn{7}{c}{$100 \%$} & & & \\
\hline
\end{tabular}


CYCB1;1 gain of function

\begin{tabular}{|c|c|c|c|c|c|c|c|c|c|c|c|c|}
\hline \multirow[b]{2}{*}{$\mathrm{APC} / \mathrm{C}$} & \multicolumn{11}{|c|}{ Attractor(s) with period 12} & \\
\hline & 1 & 1 & 1 & 0 & 0 & 0 & 0 & 0 & 0 & 0 & 0 & 1 \\
\hline KRP1 & 0 & 1 & 1 & 0 & 0 & 0 & 0 & 1 & 0 & 0 & 0 & 0 \\
\hline CYCA $2 ; 3$ & 0 & 0 & 0 & 0 & 0 & 0 & 0 & 1 & 1 & 1 & 1 & 1 \\
\hline CDKB1;1 & 1 & 1 & 1 & 0 & 0 & 0 & 0 & 1 & 1 & 1 & 1 & 1 \\
\hline CYCB1;1 & 1 & 1 & 1 & 1 & 1 & 1 & 1 & 1 & 1 & 1 & 1 & 1 \\
\hline MYB3R1/4 & 1 & 1 & 0 & 0 & 0 & 0 & 0 & 1 & 1 & 1 & 1 & 1 \\
\hline MYB77 & 0 & 0 & 0 & 0 & 0 & 0 & 1 & 1 & 1 & 0 & 0 & 0 \\
\hline $\mathrm{E} 2 \mathrm{Fe}$ & 0 & 0 & 0 & 0 & 1 & 1 & 1 & 1 & 1 & 1 & 0 & 0 \\
\hline E2Fc & 1 & 1 & 1 & 0 & 0 & 1 & 1 & 0 & 1 & 1 & 1 & 1 \\
\hline $\mathrm{E} 2 \mathrm{Fb}$ & 0 & 0 & 0 & 0 & 0 & 1 & 1 & 1 & 1 & 0 & 0 & 0 \\
\hline E2Fa & 0 & 0 & 0 & 0 & 1 & 1 & 1 & 1 & 0 & 0 & 0 & 0 \\
\hline RBR & 1 & 1 & 1 & 0 & 0 & 0 & 0 & 0 & 1 & 1 & 1 & 1 \\
\hline SCF & 0 & 0 & 0 & 0 & 0 & 0 & 1 & 1 & 1 & 1 & 1 & 1 \\
\hline CYCD3;1 & 0 & 1 & 1 & 1 & 1 & 1 & 1 & 0 & 0 & 0 & 0 & 0 \\
\hline Freq. & & & & & & & & & & & & \\
\hline
\end{tabular}


MYB3R1/4 loss of function

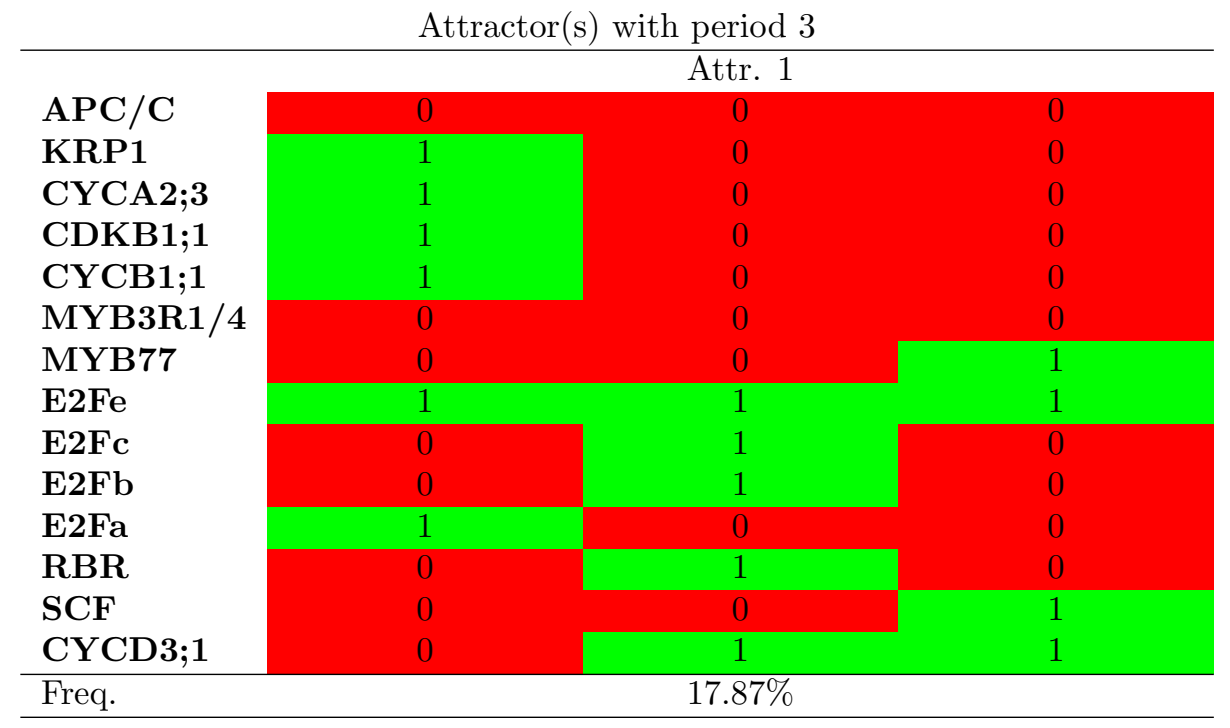

Attractor(s) with period 7

\begin{tabular}{llllllll}
\hline & & & Attr. 2 & & \\
APC/C & 0 & 0 & 0 & 0 & 0 & 0 & 0 \\
KRP1 & 0 & 0 & 0 & 0 & 0 & 1 & 0 \\
CYCA2;3 & 1 & 0 & 0 & 0 & 0 & 1 & 1 \\
CDKB1;1 & 1 & 0 & 0 & 0 & 0 & 1 & 1 \\
CYCB1;1 & 1 & 0 & 0 & 0 & 0 & 1 & 1 \\
MYB3R1/4 & 0 & 0 & 0 & 0 & 0 & 0 & 0 \\
MYB77 & 0 & 0 & 0 & 0 & 1 & 1 & 1 \\
E2Fe & 1 & 1 & 1 & 1 & 1 & 1 & 1 \\
E2Fc & 0 & 0 & 0 & 1 & 1 & 0 & 1 \\
E2Fb & 0 & 0 & 0 & 1 & 1 & 1 & 1 \\
E2Fa & 0 & 0 & 1 & 1 & 1 & 1 & 0 \\
RBR & 0 & 0 & 0 & 0 & 0 & 0 & 1 \\
SCF & 0 & 0 & 0 & 0 & 1 & 1 & 1 \\
CYCD3;1 & 0 & 1 & 1 & 1 & 1 & 0 & 0 \\
\hline Freq. & & & & $82.13 \%$ & & & \\
\hline
\end{tabular}


MYB3R1/4 gain of function

\begin{tabular}{lc|c}
\multicolumn{2}{c}{ Fixed-point attractor(s) } \\
\hline APC/C & Attr. 1 & Attr. 2 \\
KRP1 & 1 & 1 \\
CYCA2;3 & 1 & 0 \\
CDKB1;1 & 0 & 1 \\
CYCB1;1 & 1 & 0 \\
MYB3R1/4 & 0 & 1 \\
MYB77 & 1 & 0 \\
E2Fe & 0 & 0 \\
E2Fc & 0 & 1 \\
E2Fb & 1 & 0 \\
E2Fa & 0 & 1 \\
RBR & 0 & 1 \\
SCF & 1 & 0 \\
CYCD3;1 & 0 & 1 \\
\hline Freq. & 1 & $1.39 \%$ \\
\hline
\end{tabular}


MYB77 loss of function

\begin{tabular}{llllllll}
\multicolumn{7}{c}{ Attractor(s) with period 7} \\
\hline APC/C & 0 & 1 & 0 & 0 & 0 & 0 & 0 \\
KRP1 & 0 & 0 & 0 & 0 & 0 & 0 & 0 \\
CYCA2;3 & 0 & 0 & 0 & 0 & 0 & 0 & 0 \\
CDKB1;1 & 0 & 0 & 0 & 0 & 0 & 0 & 1 \\
CYCB1;1 & 0 & 0 & 0 & 0 & 0 & 0 & 1 \\
MYB3R1/4 & 0 & 0 & 0 & 0 & 0 & 0 & 0 \\
MYB77 & 0 & 0 & 0 & 0 & 0 & 0 & 0 \\
E2Fe & 0 & 1 & 1 & 1 & 1 & 1 & 1 \\
E2Fc & 0 & 1 & 0 & 1 & 1 & 0 & 1 \\
E2Fb & 0 & 1 & 0 & 1 & 1 & 1 & 1 \\
E2Fa & 1 & 1 & 1 & 1 & 1 & 1 & 1 \\
RBR & 0 & 1 & 0 & 0 & 0 & 0 & 1 \\
SCF & 0 & 0 & 0 & 0 & 1 & 1 & 1 \\
CYCD3;1 & 0 & 1 & 1 & 1 & 1 & 0 & 0 \\
\hline Freq. & & & \multicolumn{7}{c}{$100 \%$} & & \\
\hline
\end{tabular}




\section{MYB77 gain of function}

\begin{tabular}{lc} 
& Fixed-point attractor(s) \\
\hline & Attr. 1 \\
APC/C & 0 \\
KRP1 & 0 \\
CYCA2;3 & 1 \\
CDKB1;1 & 1 \\
CYCB1;1 & 1 \\
MYB3R1/4 & 1 \\
MYB77 & 1 \\
E2Fe & 1 \\
E2Fc & 1 \\
E2Fb & 0 \\
E2Fa & 0 \\
RBR & 1 \\
SCF & 1 \\
CYCD3;1 & 0 \\
\hline Freq. & $100 \%$ \\
\hline
\end{tabular}


E2Fe loss of function

\begin{tabular}{llllclll}
\multicolumn{7}{c}{ Attractor(s) with period 7 } \\
\hline APC/C & 0 & 1 & 1 & 1 & 1 & 1 & 1 \\
KRP1 & 0 & 0 & 0 & 1 & 1 & 1 & 1 \\
CYCA2;3 & 0 & 0 & 0 & 0 & 0 & 0 & 0 \\
CDKB1;1 & 0 & 0 & 0 & 1 & 1 & 1 & 1 \\
CYCB1;1 & 0 & 0 & 0 & 0 & 0 & 0 & 0 \\
MYB3R1/4 & 0 & 0 & 0 & 1 & 1 & 1 & 0 \\
MYB77 & 0 & 0 & 1 & 1 & 1 & 0 & 0 \\
E2Fe & 0 & 0 & 0 & 0 & 0 & 0 & 0 \\
E2Fc & 0 & 1 & 1 & 1 & 1 & 1 & 1 \\
E2Fb & 0 & 1 & 1 & 1 & 1 & 0 & 0 \\
E2Fa & 1 & 1 & 1 & 1 & 1 & 1 & 1 \\
RBR & 0 & 0 & 0 & 0 & 1 & 1 & 1 \\
SCF & 0 & 0 & 0 & 0 & 0 & 0 & 0 \\
CYCD3;1 & 1 & 1 & 1 & 1 & 1 & 1 & 1 \\
\hline Freq. & & \multicolumn{7}{c}{$100 \%$} & & \\
\hline
\end{tabular}


E2Fe gain of function

\begin{tabular}{lc} 
& Fixed-point attractor(s) \\
\hline & Attr. 1 \\
APC/C & 0 \\
KRP1 & 0 \\
CYCA2;3 & 1 \\
CDKB1;1 & 1 \\
CYCB1;1 & 1 \\
MYB3R1/4 & 1 \\
MYB77 & 0 \\
E2Fe & 1 \\
E2Fc & 1 \\
E2Fb & 0 \\
E2Fa & 0 \\
RBR & 1 \\
SCF & 1 \\
CYCD3;1 & 0 \\
\hline Freq. & $100 \%$ \\
\hline
\end{tabular}


E2Fc loss of function

\begin{tabular}{lc} 
& Fixed-point attractor(s) \\
\hline & Attr. 1 \\
APC/C & 0 \\
KRP1 & 0 \\
CYCA2;3 & 1 \\
CDKB1;1 & 1 \\
CYCB1;1 & 1 \\
MYB3R1/4 & 1 \\
MYB77 & 0 \\
E2Fe & 1 \\
E2Fc & 0 \\
E2Fb & 0 \\
E2Fa & 0 \\
RBR & 1 \\
SCF & 1 \\
CYCD3;1 & 0 \\
\hline Freq. & $100 \%$ \\
\hline
\end{tabular}


E2Fc gain of function

\begin{tabular}{lc} 
& Fixed-point attractor(s) \\
\hline APC/C & Attr. 1 \\
KRP1 & 0 \\
CYCA2;3 & 0 \\
CDKB1;1 & 0 \\
CYCB1;1 & 0 \\
MYB3R1/4 & 0 \\
MYB77 & 0 \\
E2Fe & 0 \\
E2Fc & 0 \\
E2Fb & 1 \\
E2Fa & 0 \\
RBR & 0 \\
SCF & 0 \\
CYCD3;1 & 0 \\
\hline Freq. & 1 \\
\hline
\end{tabular}


$\mathrm{E} 2 \mathrm{Fb}$ loss of function

\begin{tabular}{lc} 
& Fixed-point attractor(s) \\
\hline APC/C & Attr. 1 \\
KRP1 & 1 \\
CYCA2;3 & 0 \\
CDKB1;1 & 0 \\
CYCB1;1 & 0 \\
MYB3R1/4 & 0 \\
MYB77 & 0 \\
E2Fe & 0 \\
E2Fc & 0 \\
E2Fb & 1 \\
E2Fa & 0 \\
RBR & 1 \\
SCF & 0 \\
CYCD3;1 & 0 \\
\hline Freq. & 1 \\
\hline
\end{tabular}


$\mathrm{E} 2 \mathrm{Fb}$ gain of function

Attractor(s) with period 9

\begin{tabular}{|c|c|c|c|c|c|c|c|c|c|}
\hline \multirow[b]{2}{*}{$\mathrm{APC} / \mathrm{C}$} & \multicolumn{9}{|c|}{ Attr. 1} \\
\hline & 1 & 1 & 0 & 0 & 0 & 0 & 0 & 0 & 1 \\
\hline KRP1 & 0 & 1 & 0 & 0 & 1 & 0 & 0 & 0 & 0 \\
\hline CYCA $2 ; 3$ & 0 & 0 & 0 & 0 & 1 & 1 & 1 & 1 & 1 \\
\hline CDKB1;1 & 1 & 1 & 0 & 1 & 1 & 1 & 1 & 1 & 1 \\
\hline CYCB1;1 & 0 & 0 & 0 & 1 & 1 & 1 & 1 & 1 & 1 \\
\hline MYB3R1/4 & 1 & 0 & 0 & 0 & 1 & 1 & 1 & 1 & 1 \\
\hline MYB77 & 0 & 0 & 0 & 1 & 1 & 1 & 0 & 0 & 0 \\
\hline $\mathrm{E} 2 \mathrm{Fe}$ & 0 & 0 & 0 & 1 & 1 & 1 & 1 & 0 & 0 \\
\hline E2Fc & 1 & 1 & 0 & 0 & 0 & 1 & 1 & 1 & 1 \\
\hline $\mathrm{E} 2 \mathrm{Fb}$ & 1 & 1 & 1 & 1 & 1 & 1 & 1 & 1 & 1 \\
\hline E2Fa & 0 & 0 & 0 & 1 & 1 & 0 & 0 & 0 & 0 \\
\hline RBR & 1 & 1 & 0 & 0 & 0 & 1 & 1 & 1 & 1 \\
\hline SCF & 0 & 0 & 0 & 1 & 1 & 1 & 1 & 1 & 1 \\
\hline CYCD3;1 & 0 & 1 & 1 & 1 & 0 & 0 & 0 & 0 & 0 \\
\hline Freq. & \multicolumn{9}{|c|}{$100 \%$} \\
\hline
\end{tabular}


E2Fa loss of function

\begin{tabular}{lc} 
& Fixed-point attractor(s) \\
\hline APC/C & Attr. 1 \\
KRP1 & 0 \\
CYCA2;3 & 0 \\
CDKB1;1 & 0 \\
CYCB1;1 & 0 \\
MYB3R1/4 & 0 \\
MYB77 & 0 \\
E2Fe & 0 \\
E2Fc & 1 \\
E2Fb & 0 \\
E2Fa & 0 \\
RBR & 0 \\
SCF & 0 \\
CYCD3;1 & 0 \\
\hline Freq. & 1 \\
\hline
\end{tabular}


E2Fa gain of function

\begin{tabular}{lllllllll}
\multicolumn{7}{c}{ Attractor(s) with period 8 } \\
\hline APC/C & 0 & 1 & 0 & 0 & 0 & 0 & 0 & 0 \\
KRP1 & 0 & 0 & 0 & 1 & 1 & 0 & 1 & 0 \\
CYCA2;3 & 0 & 0 & 0 & 1 & 1 & 1 & 1 & 1 \\
CDKB1;1 & 0 & 0 & 0 & 1 & 1 & 1 & 1 & 1 \\
CYCB1;1 & 0 & 0 & 0 & 1 & 1 & 1 & 1 & 1 \\
MYB3R1/4 & 0 & 0 & 0 & 1 & 0 & 1 & 1 & 0 \\
MYB77 & 0 & 0 & 1 & 0 & 1 & 0 & 0 & 0 \\
E2Fe & 0 & 1 & 1 & 1 & 1 & 1 & 1 & 0 \\
E2Fc & 0 & 1 & 0 & 1 & 1 & 0 & 1 & 1 \\
E2Fb & 0 & 1 & 0 & 1 & 1 & 0 & 1 & 0 \\
E2Fa & 1 & 1 & 1 & 1 & 1 & 1 & 1 & 1 \\
RBR & 0 & 1 & 0 & 0 & 1 & 0 & 1 & 1 \\
SCF & 0 & 0 & 0 & 0 & 1 & 0 & 1 & 1 \\
CYCD3; & 0 & 1 & 1 & 1 & 1 & 0 & 1 & 0 \\
\hline Freq. & & & & \\
\hline
\end{tabular}

\begin{tabular}{lllllllllll} 
& \multicolumn{10}{c}{ Attractor(s) with period 10} \\
\hline APC/C & 1 & 1 & 0 & 1 & 0 & 0 & 0 & 0 & 0 & 1 \\
KRP1 & 0 & 1 & 0 & 0 & 0 & 1 & 0 & 0 & 0 & 0 \\
CYCA2;3 & 0 & 0 & 0 & 0 & 0 & 1 & 1 & 1 & 1 & 1 \\
CDKB1;1 & 1 & 1 & 0 & 0 & 0 & 1 & 1 & 1 & 1 & 1 \\
CYCB1;1 & 0 & 0 & 0 & 0 & 0 & 1 & 1 & 1 & 1 & 1 \\
MYB3R1/4 & 1 & 0 & 0 & 0 & 0 & 1 & 1 & 1 & 1 & 1 \\
MYB77 & 0 & 0 & 0 & 0 & 1 & 1 & 1 & 0 & 0 & 0 \\
E2Fe & 0 & 0 & 0 & 1 & 1 & 1 & 1 & 1 & 0 & 0 \\
E2Fc & 1 & 1 & 0 & 1 & 1 & 1 & 1 & 1 & 1 & 1 \\
E2Fb & 0 & 0 & 0 & 1 & 1 & 1 & 1 & 0 & 0 & 0 \\
E2Fa & 1 & 1 & 1 & 1 & 1 & 1 & 1 & 1 & 1 & 1 \\
RBR & 1 & 1 & 0 & 0 & 0 & 0 & 1 & 1 & 1 & 1 \\
SCF & 0 & 0 & 0 & 0 & 0 & 1 & 1 & 1 & 1 & 1 \\
CYCD3;1 & 0 & 1 & 1 & 1 & 1 & 1 & 0 & 0 & 0 & 0 \\
\hline Freq. & & & & & $40.48 \%$ & & & & \\
\hline
\end{tabular}


RBR loss of function

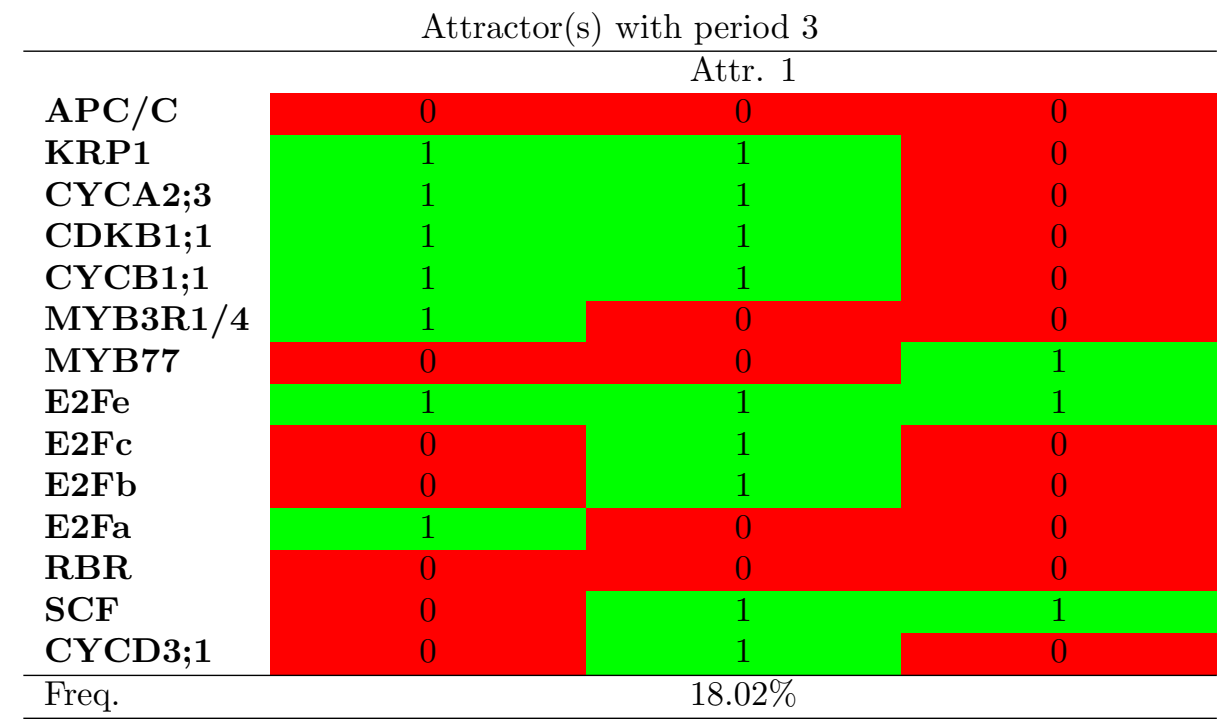

Attractor(s) with period 12

\begin{tabular}{|c|c|c|c|c|c|c|c|c|c|c|c|c|}
\hline & \multicolumn{12}{|c|}{ Attr. 2} \\
\hline $\mathrm{APC} / \mathrm{C}$ & 1 & 1 & 0 & 0 & 0 & 0 & 0 & 0 & 0 & 0 & 0 & 1 \\
\hline KRP1 & 0 & 1 & 0 & 0 & 0 & 0 & 1 & 0 & 0 & 0 & 0 & 0 \\
\hline CYCA 2;3 & 0 & 0 & 0 & 0 & 0 & 0 & 1 & 1 & 1 & 1 & 1 & 1 \\
\hline CDKB1;1 & 1 & 1 & 0 & 0 & 0 & 0 & 1 & 1 & 1 & 1 & 1 & 1 \\
\hline CYCB1;1 & 0 & 0 & 0 & 0 & 0 & 0 & 1 & 1 & 1 & 1 & 1 & 1 \\
\hline MYB3R1/4 & 1 & 0 & 0 & 0 & 0 & 0 & 1 & 1 & 1 & 1 & 1 & 1 \\
\hline MYB77 & 0 & 0 & 0 & 0 & 0 & 1 & 1 & 1 & 1 & 0 & 0 & 0 \\
\hline E2Fe & 0 & 0 & 0 & 1 & 1 & 1 & 1 & 1 & 1 & 1 & 0 & 0 \\
\hline E2Fc & 1 & 1 & 0 & 0 & 1 & 1 & 0 & 1 & 1 & 1 & 1 & 1 \\
\hline E2Fb & 0 & 0 & 0 & 0 & 1 & 1 & 1 & 1 & 0 & 0 & 0 & 0 \\
\hline E2Fa & 0 & 0 & 0 & 1 & 1 & 1 & 1 & 0 & 0 & 0 & 0 & 0 \\
\hline RBR & 0 & 0 & 0 & 0 & 0 & 0 & 0 & 0 & 0 & 0 & 0 & 0 \\
\hline SCF & 0 & 0 & 0 & 0 & 0 & 1 & 1 & 1 & 1 & 1 & 1 & 1 \\
\hline CYCD3;1 & 0 & 1 & 1 & 1 & 1 & 1 & 0 & 0 & 0 & 0 & 0 & 0 \\
\hline Freq. & \multicolumn{12}{|c|}{$81.98 \%$} \\
\hline
\end{tabular}




\section{RBR gain of function}

\begin{tabular}{lc} 
& Fixed-point attractor(s) \\
\hline APC/C & Attr. 1 \\
KRP1 & 0 \\
CYCA2;3 & 0 \\
CDKB1;1 & 0 \\
CYCB1;1 & 0 \\
MYB3R1/4 & 0 \\
MYB77 & 0 \\
E2Fe & 0 \\
E2Fc & 1 \\
E2Fb & 0 \\
E2Fa & 0 \\
RBR & 1 \\
SCF & 1 \\
CYCD3;1 & 0 \\
\hline Freq. & 1 \\
\hline
\end{tabular}


SCF loss of function

Attractor(s) with period 8

\begin{tabular}{lllllllll}
\hline & & \multicolumn{7}{c}{ Attr. 1} \\
APC/C & 0 & 0 & 0 & 0 & 0 & 0 & 0 & 0 \\
KRP1 & 0 & 0 & 0 & 0 & 1 & 1 & 1 & 1 \\
CYCA2;3 & 0 & 0 & 0 & 0 & 1 & 1 & 1 & 1 \\
CDKB1;1 & 0 & 0 & 0 & 0 & 1 & 1 & 1 & 1 \\
CYCB1;1 & 0 & 0 & 0 & 0 & 1 & 1 & 1 & 1 \\
MYB3R1/4 & 0 & 0 & 0 & 0 & 1 & 1 & 1 & 0 \\
MYB77 & 0 & 0 & 0 & 1 & 1 & 1 & 0 & 0 \\
E2Fe & 0 & 1 & 1 & 1 & 1 & 1 & 1 & 0 \\
E2Fc & 0 & 0 & 1 & 1 & 1 & 1 & 1 & 1 \\
E2Fb & 0 & 0 & 1 & 1 & 1 & 1 & 0 & 0 \\
E2Fa & 0 & 1 & 1 & 1 & 1 & 0 & 0 & 0 \\
RBR & 0 & 0 & 0 & 0 & 0 & 1 & 1 & 1 \\
SCF & 0 & 0 & 0 & 0 & 0 & 0 & 0 & 0 \\
CYCD3;1 & 1 & 1 & 1 & 1 & 1 & 1 & 1 & 1 \\
\hline Freq. & & & $100 \%$ & & & \\
\hline
\end{tabular}


SCF gain of function

\begin{tabular}{lcc}
\multicolumn{3}{c}{ Attractor(s) with period 2 } \\
\hline APC/C & 0 & Attr. 1 \\
KRP1 & 0 & 1 \\
CYCA2;3 & 0 & 0 \\
CDKB1;1 & 0 & 0 \\
CYCB1;1 & 0 & 0 \\
MYB3R1/4 & 0 & 0 \\
MYB77 & 0 & 0 \\
E2Fe & 0 & 1 \\
E2Fc & 0 & 1 \\
E2Fb & 0 & 1 \\
E2Fa & 1 & 1 \\
RBR & 0 & 1 \\
SCF & 1 & 1 \\
CYCD3;1 & 0 & 0 \\
\hline Freq. & & $100 \%$ \\
\hline
\end{tabular}


CYCD3;1 loss of function

\begin{tabular}{lcc}
\multicolumn{3}{c}{ Attractor(s) with period 2 } \\
\hline APC/C & 0 & Attr. 1 \\
KRP1 & 0 & 1 \\
CYCA2;3 & 0 & 0 \\
CDKB1;1 & 0 & 0 \\
CYCB1;1 & 0 & 0 \\
MYB3R1/4 & 0 & 0 \\
MYB77 & 0 & 0 \\
E2Fe & 0 & 1 \\
E2Fc & 0 & 1 \\
E2Fb & 0 & 1 \\
E2Fa & 1 & 1 \\
RBR & 0 & 1 \\
SCF & 0 & 0 \\
CYCD3;1 & 0 & 0 \\
\hline Freq. & & $100 \%$ \\
\hline
\end{tabular}


CYCD3;1 gain of function

\begin{tabular}{lc} 
& Fixed-point attractor(s) \\
\hline APC/C & Attr. 1 \\
KRP1 & 0 \\
CYCA2;3 & 0 \\
CDKB1;1 & 1 \\
CYCB1;1 & 1 \\
MYB3R1/4 & 1 \\
MYB77 & 1 \\
E2Fe & 0 \\
E2Fc & 1 \\
E2Fb & 0 \\
E2Fa & 0 \\
RBR & 0 \\
SCF & 0 \\
CYCD3;1 & 1 \\
\hline Freq. & 1 \\
\hline
\end{tabular}

